\title{
URBAN REGENERATION PROGRAMS FOR SUSTAINABLE PLANNING IN HIGHLY VULNERABLE URBAN CONTEXTS
}

DOI: http://dx.doi.org/10.18509/GBP.2016.34

UDC: 332.146.2:711.2(450)

\author{
Dr. Riccardo Privitera \\ Dr. Daniele La Rosa \\ Department of Civil Engineering and Architecture, University of Catania, Italy
}

\begin{abstract}
Topics of sustainable urban development and environmental sustainability are worldwide considered as fundamental for every strategy of urban transformation, renewal and regeneration. In particular, urban regenerations are urban re-development programs involving the rebirth or renewal of selected urban areas or district that have faced periods of decline due to compounding and intersecting pressures. The programs cover many aspects of the area to be re-generated such as physical, social and environmental contexts. Re-use of already built up areas and buildings, reduction of the demand for new soils to be developed, increasing of appealing of dense city areas, increasing of social and spatial resilience are among the positive consequences of these programs.

However, in the current debate about urban regeneration, few studies have evaluated the real environmental outcomes and effectiveness of regeneration programs in terms of physical variables such as new provided greenspaces, accessibility to public transportation, climate change or seismic risk reduction. This paper proposes a method to quantify the real outcomes and effectiveness of urban regeneration programs with reference to the above mentioned variables.

As a real experience of urban planning, the new Local Spatial Plan for the Municipality of Catania, a medium sized city in Southern Italy, is presented. The city is characterised by a high density urban fabric, a general lack of urban greenspaces and high levels of traffic congestion due to a massive use of private transportation. The urban fabric is also very vulnerable to seismic and climate change risks. Among the transformation tools, the new Local spatial plan proposes regeneration actions aimed at the complete regeneration of old and dilapidated areas, not classified as historical heritage and heavily vulnerable to seismic risk. These actions include the complete demolition and reconstruction of these areas within clearly defined boundaries, contributing to minimise soil consumption, maintaining as open public spaces the majority of existing non urbanised areas within the densely built-up settlement. The program of regeneration can dramatically contributes to the reduction of seismic and climate change risk and achieve a general requalification of the urban environment.

Starting from this planning experience, this paper focus on the evaluation of the regenerations programs included in the Local Spatial Plan. Regeneration Areas (RAs) have been identified by the municipality as characterized by high level of seismic vulnerability, urban degradation, lack of public services and urban environment quality. For the chosen areas, this study proposes the evaluation of the transformations potentially occurring in the urban context by the proposed regeneration program. The following aspects are evaluated:

- reduction of risks (in terms of exposition and vulnerability to seismic and climate change related risks)
\end{abstract}


- mobility (concerning the presence of public means of transports, distance to the transit stops, roads, pedestrian and cycling lanes)

- accessibility increase (in terms of access to trip attractions)

- land-use diversity (in terms of number and distribution of different land uses)

- public spaces and services (in terms of extension and functions)

Each of the aforementioned aspects are evaluated by spatial indicators calculated by GIS. All indicators are calculated at different and size increasing units, in order to understand the effect of a single regeneration project and of a number of concurrent projects within the considered geographical units. The geographical units are the RAs and districts. Different combinations of regeneration projects will be thus evaluated to highlight which projects produce the most relevant effects, calculated with the proposed indicators.

This will allow the municipality to define scenarios of regeneration priority, in terms of which projects might be financed and implemented firstly because of their higher positive effect on the urban environment. Such scenarios will generate positive effects not only to the single areas to be regenerated but also to wider urban contexts, significantly reducing the urban vulnerability to seismic and climate change risks and at the same time producing more livable and healthy urban environment.

Keywords: urban regeneration, sustainability, urban planning, urban vulnerability, risk reduction.

\section{URBAN REGENERATION AND SUSTAINABILITY. A NEW APPROACH FOR DESIGNING CITIES}

An increasing body of literature highlights the role of sustainability approaches for the definition of policies for urban transformation [1]. Particularly, urban regeneration is often considered as one of the most effective mechanism for the delivery of sustainable urban development. Examples of planning strategies for the contemporary sustainable city include the re-use of urban dismissed land and abandoned buildings. As a direct consequence, this can reduce soil sealing, decrease the demand of new developments in periurban areas and increase the appealing of urban centers [2].

Urban regeneration programs have been recently developed across Europe as a holistic strategy to achieve urban quality through challenging social inequity and blight of urban spaces. These programs are characterized by a very high level of complexity of economic, social, political and environmental processes and are based on urban development policies involving the re-building or re-configuration of urban spaces [3].

In this respect, urban regeneration is different from urban renewal, because the latter refers to the physical aspects of urban transformations, without any attempt of addressing the physical, social and economics inequalities of disadvantaged neighborhoods.

Due to the different options in terms of types of interventions on built-up areas, regeneration policies need to be specified according to the physical, social, cultural and economic features of the urban areas [4], as well as the urban development model adopted by local administrations.

Particularly, urban regeneration programs oriented to urban sustainability and energy efficiency must be implemented at local urban or regional scale. The objectives of this kind of programs are aimed at increasing the energy performance and anti-seismic response of existing urban fabric, enhancing the provision of public services and the related ecosystem services [5]. The scale of regeneration programs is a fundamental issue 
for their effectiveness: at urban scale, interrelations among different components of urban fabric can be taken into account in order to achieve high levels of urban environment quality. Built-up areas density, land-use types, and mobility represent some of the features that should be considered when developing sustainable planning strategies [6].

However, most of the European and Italian urban regeneration practices have showed a sectorial approach. They are often planned with little relation to municipality local spatial plans, involve limited areas at neighborhood or district level and are not developed according to sustainable planning models [7]. Considering urban regenerations as a part of a broader urban development plan could help to overcome such a sectorial approach and provide benefits for the entire urban context and lead to a new approach for designing cities aimed at dealing with regeneration programs as an opportunity to sustainable implement urban transformation strategies.

Despite the consolidated and central role of urban regenerations programs for planning sustainable cities, few studies have evaluated the real environmental outcomes and effectiveness of regeneration programs in terms of physical variables such as new provided greenspaces, accessibility to public transportation, climate change or seismic risk reduction. For these reason, this paper argues on the efficacy of regenerations programs and proposes a method to quantify the related outcomes with reference to the abovementioned environmental and urban variables. The work focuses on a real planning practice, the new Local Spatial Plan of the city of Catania (Italy). Among the different land transformation mechanisms provided by the Local Spatial Plan, several regeneration programs are included, especially in peripheral areas of the city, mainly characterized by environmental, urban and social forms of blight. For these areas, this study proposes the evaluation of the transformations potentially occurring in the urban context by the proposed regeneration program. The following aspects are evaluated:

- reduction of seismic risk (in terms of exposition to seismic risk);

- accessibility increase (in terms of access to trip attractions);

- land-use diversity (in terms of number and distribution of different land uses)

Each of the aforementioned aspects is evaluated by spatial indicators calculated by GIS. All indicators are calculated at different and size increasing units, in order to understand the effect of a single regeneration project and of a number of concurrent projects within the considered geographical units.

\section{METHOD AND RESULTS}

As previously, this study evaluates the transformations potentially occurring in the urban context by the proposed regeneration program. The chosen model is based on spatial indicators calculated by GIS that allow to evaluate the main regeneration actions in terms of benefits on specific urban areas. Six RAs have been chosen among the total 49 RAs provided by the program. These areas are located at the west urban periphery of the municipality of Catania. The selected district is mainly characterized by residential land uses, buildings with low levels of performance in terms of energy efficiency and antiseismic response, and lack of public greenspaces and services (fig. 1).

The effects of regeneration program have been evaluated within geographic units that include buffers of $500 \mathrm{~m}, 1000 \mathrm{~m}$ and $2000 \mathrm{~m}$ generated from the geometrical centroid of the set of the 6 RAs (Fig. 1). The increasing distance buffers aim at understanding how the effects of the 6 RAs change when larger territorial units are taken into account. The 
widest buffer covers an area of $12 \mathrm{~km}^{2}$ that represents about a sixth of the total municipality area.

Within these buffers the following urban regeneration positive effects are evaluated, as anticipated in previous section:

- reduction of seismic risk (in terms of exposition);

- increase of accessibility (in terms of access to urban functions);

- increase of the mix of land uses (in terms of number and diversity of land-use types).

One GIS-based indicator has been introduced for each of the three effects.

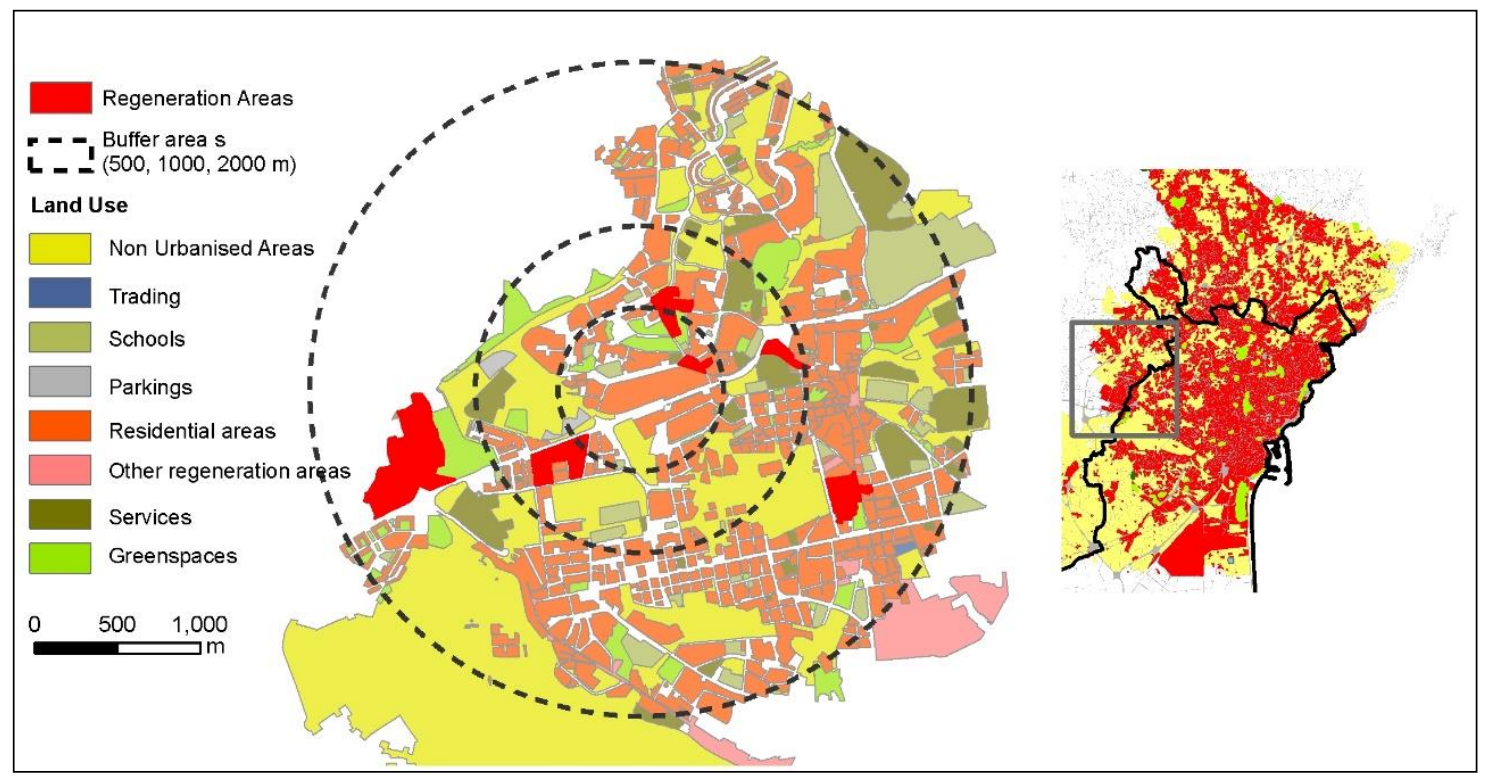

Figure 1 - Selected regeneration areas and buffer areas in the municipality of Catania Reduction of seismic risk exposition

This indicator evaluates the reduction of the population exposition to seismic risk as a positive effect of the regeneration actions [8]. It is calculated for each of the three considered buffers as the population that is not exposed to seismic risk with the following formula:

$$
1 \text { - (PopE0-PopEr)/PopT, }
$$

where PopE0 represents the current population under potential risk, PopEr represents the new population exposed after the implementation of the regeneration actions and PopT is the total population of the RA.

The indicator is calculated by GIS taking into account the number of residents of the census tracts that are within the buffers (as showed in Fig. 2 for the 500 m radius buffer). Tab. 1 shows as the percentage of the population under risk exposition linearly decreases with the increasing of buffer area. Moreover, the indicator score does not change substantially when moving from the 500 buffer to the $1000 \mathrm{~m}$ buffer: this means that population not exposed to seismic risk after the regeneration action does not vary until the considered buffer size of $1000 \mathrm{~m}$. 
Table 1. - Reduction of population exposed to seismic risk after the RA

\begin{tabular}{ccccc}
\hline Buffer-Area & PopE & PopEr & PopT & RSRE \\
\hline Buffer 500 m & 5002 & 201 & 5166 & 0.07 \\
Buffer 1000 m & 21416 & 1836 & 22164 & 0.12 \\
Buffer 2000 m & 72353 & 3332 & 73354 & 0.06 \\
\hline
\end{tabular}

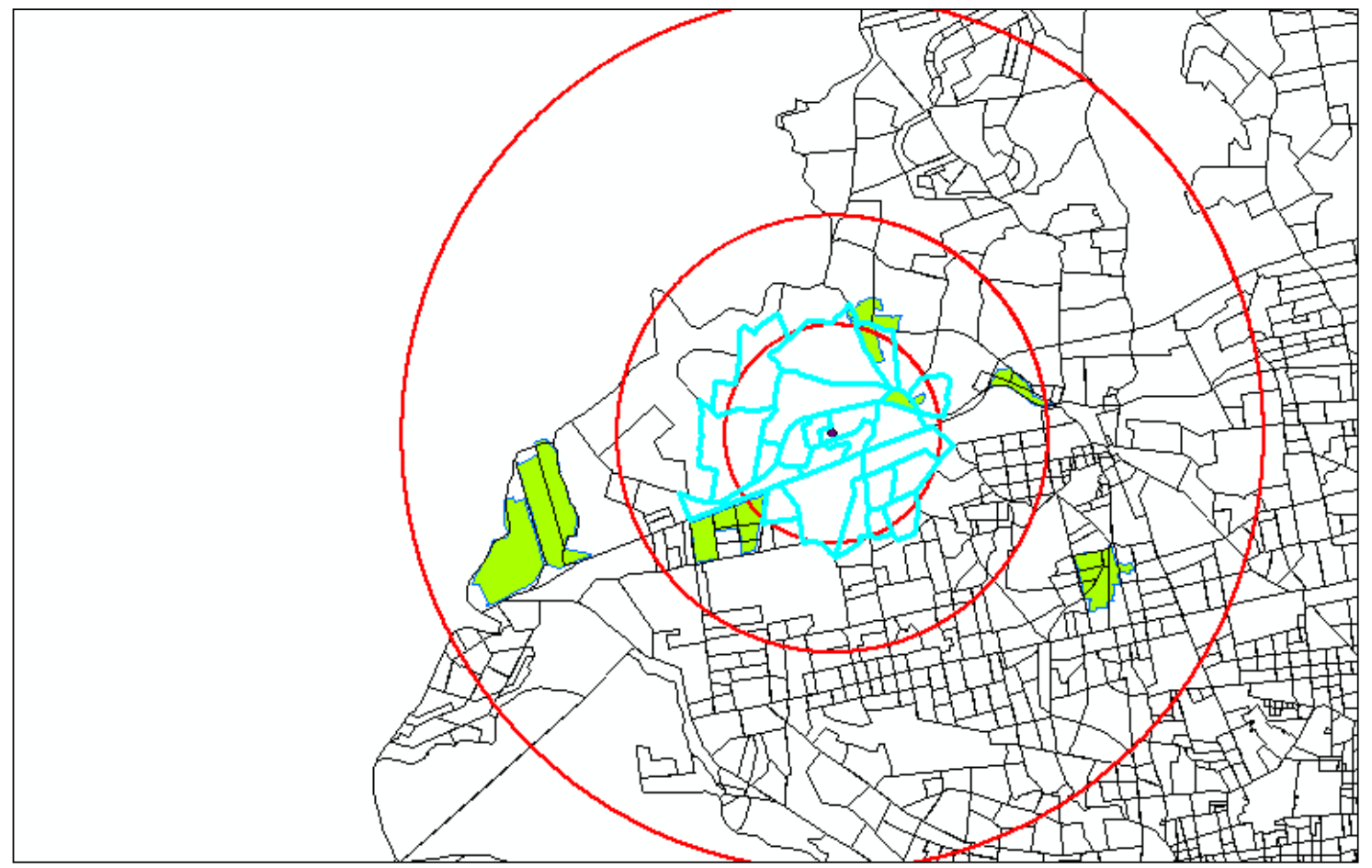

Figure 2. - Selected census tracts within the study area.

Increase of accessibility to urban functions

The indicator evaluates the number of residents that can access to the urban functions introduced by the regeneration program within each RA. It is calculated by taking into account two different accessibility conditions: a 300 meter radius for pedestrian trips and a 1500 meter radius for bicycle trips (Fig. 3). Census tracts that are located within these units are selected with a geographical query and then intersected with the 3 buffers of different size (Fig. 3). This allows to evaluate the number of residents in the census tracts that could access to the RAs that are within $300 \mathrm{~m}$ and $1500 \mathrm{~m}$ distance and are present at the same time within one of the three buffers [9]. Fig. 3 shows an example of the method referred to the case of $300 \mathrm{~m}$ unit (pedestrian mobility-walking distance) within the $500 \mathrm{~m}$ buffer. Results are showed in Tab. 2.

Table 2. Population with accessibility to new urban functions in RA, for the 3 buffer and travel behavior

\begin{tabular}{ccc}
\hline Buffer Areas & Pop in 300 m buffer (walking distance) & Pop in 1500 m buffer (bike distance) \\
\hline Buffer 500 m & 3397 & 4553 \\
Buffer 1000 m & 9579 & 17394 \\
Buffer 2000 m & 23438 & 65234 \\
\hline
\end{tabular}




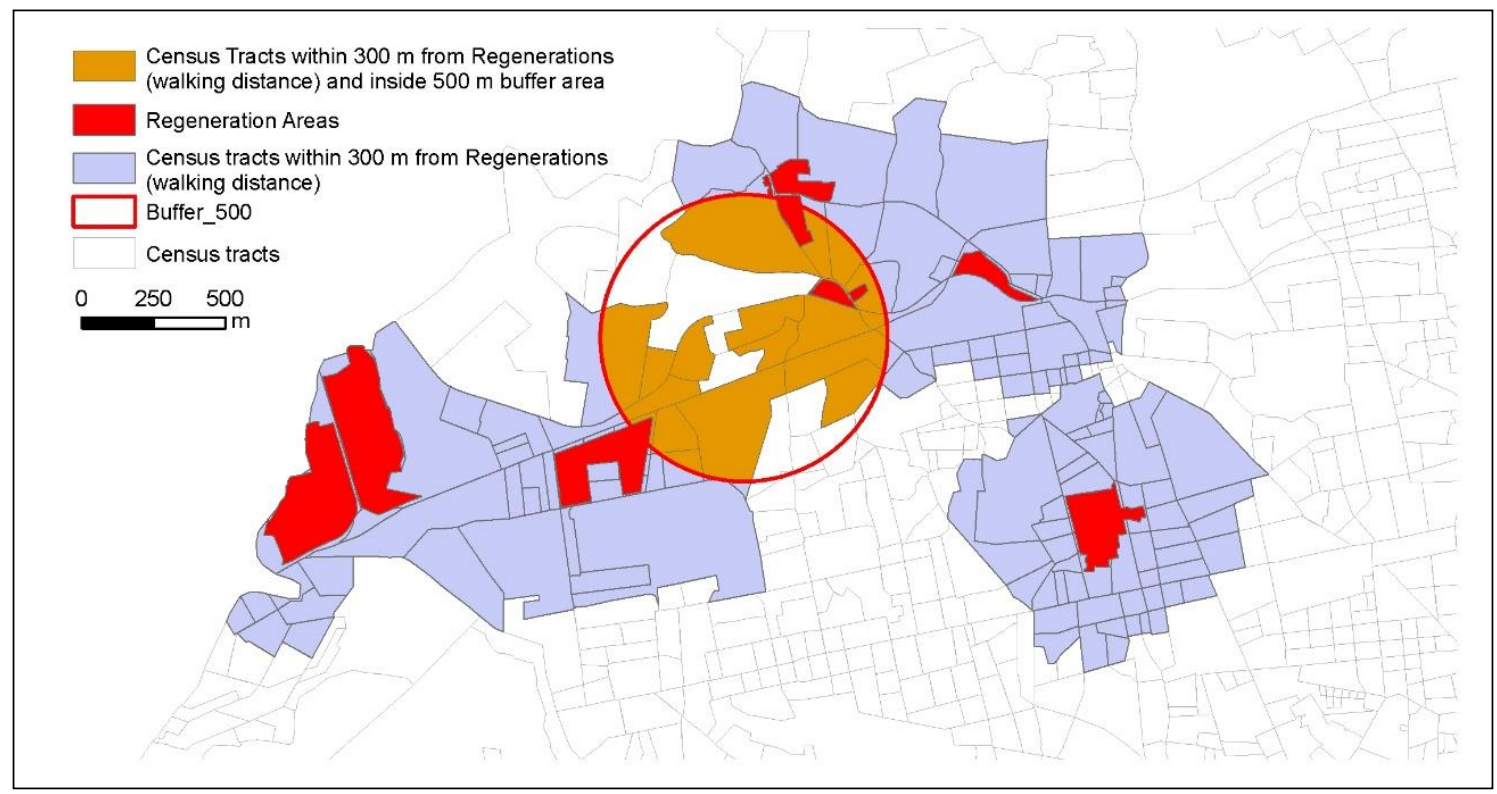

Figure 3. Accessibility modeling for pedestrian mobility

\section{Increase of mixed land uses}

Regeneration actions take place in urban fabrics mainly characterized by residential land uses. The increase of the mix of land uses provided by the regeneration program has been quantified within the three buffers either in terms of total number of land-use types and diversity. The latter evaluates using the Shannon-Weaver entropy index [10], that is widely applied in spatial analysis of land uses distribution and diversity assessment [11].

Table 4. - Increase of mixed land uses

\begin{tabular}{cccccc}
\hline Buffer & $\begin{array}{c}\text { \# land uses } \\
\text { (current) }\end{array}$ & $\begin{array}{c}\text { Shannon } \\
\text { Diversity } \\
\text { (current) }\end{array}$ & $\begin{array}{c}\text { \# land uses (after } \\
\text { regenerations) }\end{array}$ & $\begin{array}{c}\text { Shannon } \\
\text { Diversity (after } \\
\text { regenerations) }\end{array}$ & $\begin{array}{c}\text { Shannon's } \\
\text { increase (\%) }\end{array}$ \\
\hline Buffer 500 m & 5 & 1.114 & 7 & 1.170 & 0.05 \\
Buffer 1000 m & 6 & 1.293 & 8 & 1.412 & 0.08 \\
Buffer 2000 m & 7 & 1.450 & 10 & 1.497 & 0.03 \\
\hline
\end{tabular}

Fig. 4 shows land uses configuration before and after the regeneration program. As shown by indicators' scores in Tab. 3, the wider the geographic unit is the higher is the diversity value (the highest increase of diversity score can be seen for the $1000 \mathrm{~m}$ radius buffer). 


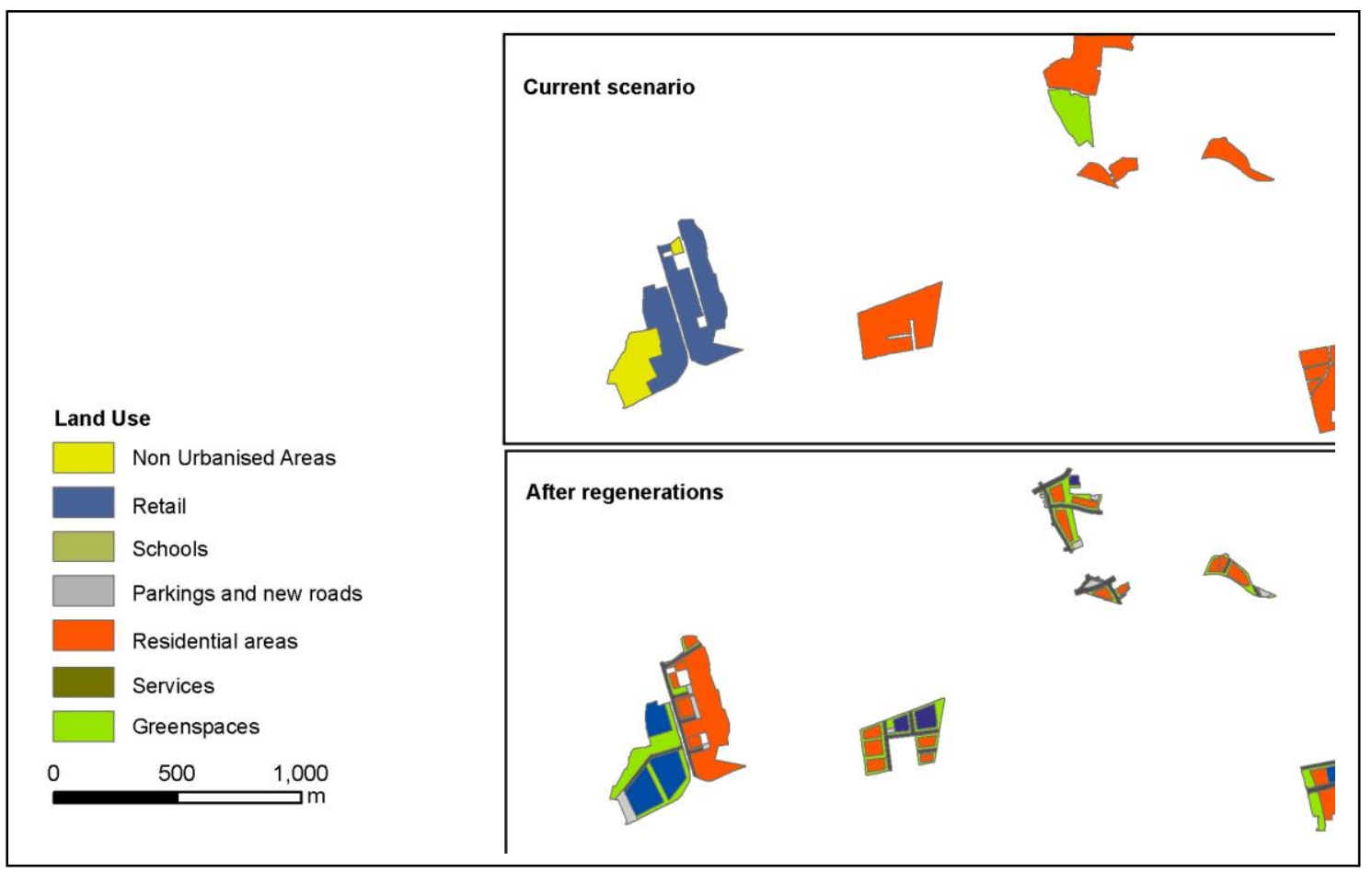

Figure 4. Land uses configurations before and after regenerations

\section{DISCUSSIONS AND CONCLUSIONS}

Overcoming the traditional development strategy, urban regeneration programs are a promising approach to re-think blighted urban areas through actions aimed reducing exposition to seismic risk, energy consumption of the buildings, mitigating climate change effects and safeguarding existing non urbanized areas within urban fabric. Urban regeneration programs strictly intertwined to the municipality local spatial plan at urban scale ensures the public control of the development process and high urban quality levels of the interventions. However, the positive effects of these programs involve areas that are wider than the Regeneration Areas and have an influence in a relevant portion of the city. This means that these effects need to be evaluated with proper tools and indicators in order to understand which of the RAs produce the most relevant effects on the entire urban context.

First results of the proposed indicators confirm that urban regeneration benefits provided by RAs are extended to contiguous areas, proxied in the method by increasing size buffers. Indicators' scores show how these benefits do not decrease substantially when increasing the area of the buffer from 500 to $1000 \mathrm{~m}$ and remain significant even when the wider buffer of $2 \mathrm{~km}$ is taken into account. In order to have a more detailed evaluation of the benefits of Regeneration Programs, other indicators can be added in the method such as mobility indicators concerning the presence of public means of transports, distance to the transit stops, roads, pedestrian and cycling lanes, and socio-economic factors influencing residents' behavior [12]. Furthermore, the method can also be improved by evaluating the concurrent effects of different combinations of RAs: this will allow the municipality to define scenarios of regeneration priority, in terms of which projects might be financed and implemented firstly because of their higher positive effect on the urban environment. Such scenarios will generate positive effects not only to the single areas to be regenerated but also to wider urban contexts [8], significantly reducing 
the urban exposure to seismic and climate change risks and at the same time producing more livable and healthy urban environment.

\section{REFERENCES}

[1] Newman, P., Jenning, I. Cities as sustainable ecosystem, Principles and Practices, Washington DC, 2008.

[2] Couch, C., Dennemann, A. Urban Regeneration and sustainable development in Britain, Cities, vol. 17, no. 2, pp. 137-147, 2000.

[3] Paddison, R. Housing and Neighbourhood Quality: Urban Regeneration, International Encyclopedia of Housing and Home, pp 288-293, 2012.

[4] Kleinhans, RJ. Housing policies and regeneration, International Encyclopedia of Housing and Home, pp 590-595, 2012.

[5] Privitera, R., Martinico, F., La Rosa, D., Pappalardo, V. The role of non-urbanized areas for designing an Urban Green Infrastructure. A proposal for an Urban Green Infrastructure in a medium sized city in southern Italy, Nordic Journal of Architectural Research 25 (2), pp 157-186, 2013.

[6] Callender, J. Sustainable Urban Development, International Encyclopedia of Housing and Home, pp 129-133, 2012.

[7] Zheng, H.W., Shen, G.Q.P., Wang, H. A review of recent studies on sustainable urban renewal, Habitat International 41, pp 272-279, 2014.

[8] Güzey, O. The last round in restructuring the city: Urban regeneration becomes a state policy of disaster prevention in Turkey, Cities 50, pp 40-53, 2016.

[9] La Rosa, D., 2014. Accessibility to greenspaces: GIS based indicators for sustainable planning in a dense urban context, Ecological Indicators 42, pp 122-134.

[10] Shannon, C. E. A mathematical theory of communication. The Bell System, Technical Journal 27, pp 379-423 and pp 623-656, 1948.

[11] Yoshida, T., Tanaka, K. Land-use diversity index: a new means of detecting diversity at landscape level, Landscape and Ecological Engineering November 1, pp 201-206, 2005.

[12] Laprise, M., Lufkin, S., Rey, E. An indicator system for the assessment of sustainability integrated into the project dynamics of regeneration of disused urban areas, Building and Environment 86, pp. 29-38, 2015. 